\section{Classification of Kentucky Bluegrass Genotypes Grown as Spaced-plants}

\author{
Stacy A. Bonos ${ }^{1}$, William A. Meyer ${ }^{2}$, and James A. Murphy ${ }^{3}$ \\ Department of Plant Science, Rutgers University, New Jersey Agricultural \\ Experiment Station, 59 Dudley Road, Foran Hall, New Brunswick, \\ NJ 08901-8520
}

\section{Additional index words. Poa pratensis, growth habit, cultivar}

\begin{abstract}
The apomictic breeding behavior of Poa pratensis L. provides an opportunity to study many unique genotypes that can vary dramatically in characteristics such as disease resistance, stress tolerance and growth habit. The classification of Kentucky bluegrass into types is based on common growth and stress performance characteristics gathered from field turf trials. These classification types include the Compact, Bellevue, Mid-Atlantic, BVMG ('Baron, 'Victa', 'Merit', and 'Gnome'), Common, and Aggressive types. A spaced-plant nursery trial was established in May 1996 to quantify morphological and growth characteristics of 45 cultivars and selections representing the major types of Kentucky bluegrass. Plant height, panicle height, flag leaf height and length, subtending leaf length and width, rhizome spread, and longest extending rhizome were measured 10 days after anthesis. Compact type cultivars had a lower, more prostrate growth habit than the Common, Mid-Atlantic, and Bellevue types. Mid-Atlantic type cultivars had a wider rhizome spread than Compact type cultivars. Principal component analysis of morphological measurements made on spaced-plants supports the classification types of the Common, Compact, Bellevue, Mid-Atlantic, and BVMG, but not necessarily the Aggressive type.
\end{abstract}

The apomictic breeding behavior of Kentucky bluegrass contributes to the development of many unique genotypes (Meyer and Funk, 1989). Before 1970, selection among and within populations of natural ecotypes was the only method that had produced commercially successful cultivars (Bashaw and Funk, 1987). Since that time, improvements in hybridization techniques (Pepin and Funk, 1971) and better selection of parents have enhanced the species. A large number of these genotypes of Kentucky bluegrass have been classified into groups based on growth and performance characteristics (Bara et al., 1993; Murphy et al., 1997). They include Compact, Bellevue, Mid-Atlantic, BVMG (named after 'Baron', 'Victa', 'Merit', and 'Gnome'), Common and Aggressive types.

Murphy et al. (1997) evaluated the classification of Kentucky bluegrass genotypes managed as mowed turf plots and reported on turf characteristics, including leaf spot disease caused by Dreshlera poae (Baudys) Shoemaker, stripe smut disease incited by Ustilago striiformis (Westend.) Niessl., winter color and dormancy, and tolerance to and recovery from summer stress. The Aggres-

\footnotetext{
Received for publication 21 June 1999. Accepted for publication 10 Nov. 1999. Research supported by the Rutgers Center for Turfgrass Science, New Jersey Agricultural Experiment Station, and New Jersey Turfgrass Association Publication. No. D12264-10-99. The cost of this paper was defrayed in part by the payment of page charges. Under postal regulations, this paper must therefore be hereby marked advertisement soley to indicate this fact. ${ }^{1}$ Graduate Research Assistant. E-mail address: bonos@eden.rutgers.edu

${ }^{2}$ Professor.

${ }^{3}$ Assistant Professor.
}

sive type includes a diverse group of cultivars that vary in many characteristics but form a group based on their strong lateral growth habit and high shoot density. Cultivars of this type can dominate other species or cultivars when used in blends or mixtures. The Bellevue type exhibits excellent cool-season vigor, remaining green into the winter and initiating early spring green-up (Bara et al., 1993; Murphy et al., 1997). The BVMG type, named after the first commercially released cultivars of this group ('Baron', 'Victa', 'Merit', and 'Gnome'), has a high seed yield potential, but has become increasingly susceptible to stripe smut disease (Bara et al., 1993; Morris, 1997; Murphy et al., 1997). Compact type cultivars are characterized by a low, compact growth habit and exhibit good to excellent resistance to leaf spot diseases incited by Dreshlera and Bipolaris sp. (Bara et al., 1993; Murphy et al., 1997). Mid-Atlantic ecotypes form a vigorous turf of medium-high density. This group has good performance during summer stress and good recuperative ability (Bonos, 1997). The Common type is characterized as having an erect growth habit and narrow leaf blades (Burt and Christians, 1990). This group is susceptible to leaf spot disease but can exhibit good summer stress tolerance (Bara et al., 1993; Murphy et al., 1997). Cultivars in this type are typically used for low maintenance turfs, permanent pastures, or conservadisease is more severe (Meyer and Funk, 1989). Some Kentucky bluegrass genotypes exhibit characteristics intermediate between two or more groups and are currently categorized in the Other type.

The objective of this study was to quantify morphological and growth characteristics of tion areas in humid regions where leaf spot the major types of Kentucky bluegrass grown as spaced-plants.

\section{Materials and Methods}

Plant material. Forty-three cultivars and selections (Table 1) were grown for 5 months under greenhouse conditions from seed acquired from the 1990 Kentucky bluegrass national test coordinated by the National Turfgrass Evaluation Program (NTEP). The plants were then transplanted to a spacedplant ( $0.6 \mathrm{~m}$ between plants) nursery trial established 14 May 1996 on a Freehold sandy loam (fine, mixed, mesic, Typic Hapludult), using a randomized complete-block design with nine replications. Mowing was withheld to allow inflorescence development and anthesis; however, the plants were mowed seasonally after data collection and before seed maturity. Fertilizer $(34 \mathrm{~N}-0 \mathrm{P}-0 \mathrm{~K})$ was applied in $1996\left(7.6 \mathrm{~g} \cdot \mathrm{m}^{-2}\right.$ on $16 \mathrm{Sept} ., 7$ Oct., and 6 Nov.) and 1997 (7.6 g.m $\mathrm{m}^{-2}$ on 13 Oct. and $5.2 \mathrm{~g} \cdot \mathrm{m}^{-2}$ on 24 Oct.). No fertilizer was applied in 1998. The plants were not irrigated. Labeled pre- and postemergence turf herbicides were used to control broadleaf weeds and Poa annua L.

Morphological measurements. Measurements were taken from 28 May to 6 June 1997 and from 3 June to 8 June $1998, \approx 10$ d after anthesis, on the parameters specified in the U.S. Dept. of Agriculture (USDA) Plant Variety Protection Application Form for Kentucky bluegrass (Beltsville, Md). Plant height (one measurement per plant) was measured from the soil surface to the top of the third tallest panicle. The lengths of five panicles per plant (from the flag leaf to the top of the panicle) were recorded, and the heights of five flag leaves per plant were measured from the soil surface to the collar of the leaf. These three measurements were used

Table 1. Kentucky bluegrass cultivars and selections evaluated in a spaced-plant nursery established in May 1996.

\begin{tabular}{lc}
\hline Classification type & Cultivar or selection \\
\hline Aggressive & A-34, Touchdown, \\
Limousine & Georgetown, Suffolk, \\
Bellevue & Freedom, Banff \\
Compact & J-1555*, Quantum Leap, \\
& Liberator, America, \\
& Unique, Moonlight, \\
& Glade, Amazon, Nugget, \\
& Able-1, Blacksburg \\
& South Dakota Certified, \\
& Kenblue, Huntsville \\
Baron, Gnome, Kelly, \\
Merit \\
Mommon & Preakness, Livingston, \\
BVMG & A84-563 VT (VP)*, \\
Mid-Atlantic & Wabash, Bel 21*, \\
& Baltimore City*, \\
& A82-1167 VT*, \\
Riverside Park (RSP)*, & ZPS-204 VT*, \\
& Broadway, Washington, \\
& H86-697*, Nublue, Ram I \\
&
\end{tabular}

*Denotes experimental selection 
to identify low, prostrate growth compared with erect, upright growth. The blade lengths of three flag leaves (from the ligule to the leaf tip) and of three of the first leaves subtending the flag leaf were recorded on each plant. The widths of three subtending leaves per plant were measured at the widest point of the leaf. Flag leaf length, subtending leaf length, and subtending leaf width measurements were chosen randomly from the plants using the same leaf for length and width measurements, and used to characterize those types with distinct leaf texture differences, such as narrow leaf blades. Rhizome length (two rhizomes per plant) was taken as the farthest spread (1-year and 2-year spread) from the center of the plant to the outside edge. Three of the longest extending rhizomes were measured. The tiller farthest from the center of the plant was excavated to expose the rhizome, which was measured to its end or to the node of the closest tiller. Rhizome measurements were used to characterize aggressive type cultivars and Mid-Atlantic ecotypes that have been described as having an extensive rhizome system.

Data for each entry were combined to develop means for the representative type (Table 1) and subjected to analysis of variance (ANOVA). Means were separated using Fisher's protected least significant difference (LSD).

Principal component analysis. The relationships between cultivars based on all morphological characteristics were tested by subjecting the data to multivariate principal component analysis (PCA) PROC PRINCOM (SAS Institute, 1985). Forty-three cultivars and eight morphological variables were used to complete the analysis.

PCA restructures the data set containing many correlated variables into smaller sets of components of the original variables, which can be scored along independent linear axes. The analysis can reveal clusters of morphologically similar phenotypes when the data are plotted against major principal components (Iezzoni and Pritts, 1991).

The original variables (in this case plant height, panicle length, flag leaf length, etc.) are transformed to create new variables and axes that account for more variation in the original data than any single variable alone. The first transformation equals the first principal component (PC1), which corresponds to the major axis or vector. New variables or principal components (equal to the number of original variables) are computed by following a transformation equation described by Iezzoni and Pritts (1991). Eigenvalues (total variance accounted for by each equation) that are $>1.0$ and account for most of the variation in the data theoretically have more information than any one single variable, and are generally used in interpretation (Iezzoni and Pritts, 1991).

\section{Results and Discussion}

All morphological measurements except flag leaf height indicated greater separation between Kentucky bluegrass types in 1997 than in 1998, perhaps because of plant age and/or interplant competition. Measurements in 1997 and 1998 indicated that Compact type cultivars had the lowest plant height and shortest panicles of all types except the BVMG type (Table 2).

The BVMG type was characterized as having a medium-low growth habit with stiff, upright seed heads and proportionally less leafiness (Bara et al., 1993). Cultivars of this type had a low growth habit similar to the Compact type cultivars (Table 2). The BVMG and Compact types had the lowest flag leaf height in 1997; the Aggressive type was similar to the Compact type in 1997 and to the BVMG type in 1998 (Table 2).

The Mid-Atlantic type has been described as having extensive rhizomes and a more upright growth habit than did Compact or BVMG types (Bara et al., 1993). The MidAtlantic ecotype cultivars had a wider rhizome spread than did Compact and Aggressive types in both 1997 and 1998 (Table 2). Flag leaf lengths of the Mid-Atlantic type were longer than those of all other types in 1997, and were similar to those of the Common type in 1998 (Table 3). Subtending leaf length was longer in the Mid-Atlantic type than in Aggressive, Compact, BVMG or Bellevue types in 1997 and 1998 (Table 3); values for the Common type were similar to those of the Mid-Atlantic type (Table 3).

The Bellevue type has been described as having a medium growth habit (Bara et al., 1993; Murphy et al., 1997). Height measurements from both 1997 and 1998 indicated that Bellevue types had a growth habit similar to the Mid-Atlantic ecotype, taller than the low, compact growth of the Compact and BVMG types, and lower than the erect growth habit of the Common types (Table 2).

Common types had the highest plant height of all types in both 1997 and 1998 (Table 2). Subtending leaf widths were narrower in the Common type than in Bellevue, Mid-Atlantic and BVMG types in 1997, and in Bellevue and Mid-Atlantic types in 1998 (Table 3). These results are supported by the findings of Ebdon and Petrovic (1998), who found that 'Kenblue' and 'South Dakota Certified' had among the narrowest leaf blades and longest leaf lengths.

Bara and coworkers (1993) described Aggressive type cultivars as having a vigorous lateral spread. The Aggressive type had longer rhizomes than all other types except the Mid-Atlantic type (Table 2).

Principal component 1 (PC1) and Principal component 2 (PC2) in 1997 accounted for $64.7 \%$ and $15.1 \%$ of the variation, respectively, or $79.8 \%$ of the total variation (Table 4). Principal component 3 raised the total variation to $88.8 \%$ but did not change the overall pattern of the graph, and therefore is not presented. PC1 and PC2 in 1998 accounted for $57.1 \%$ and $14.3 \%$ of the variation, respectively, or $71.4 \%$ of the total variation. PC3 raised the total variation to $79.8 \%$ but did not change the overall pattern and is not presented. The PC1 was best correlated with (listed in decreasing order) plant height, flag leaf height, and subtending leaf length (1997 and 1998), PC2 with flag leaf length and

Table 2. Plant height, panicle height, flag leaf height, rhizome spread, and longest rhizome of Kentucky bluegrass types established in a field trial in May 1996.

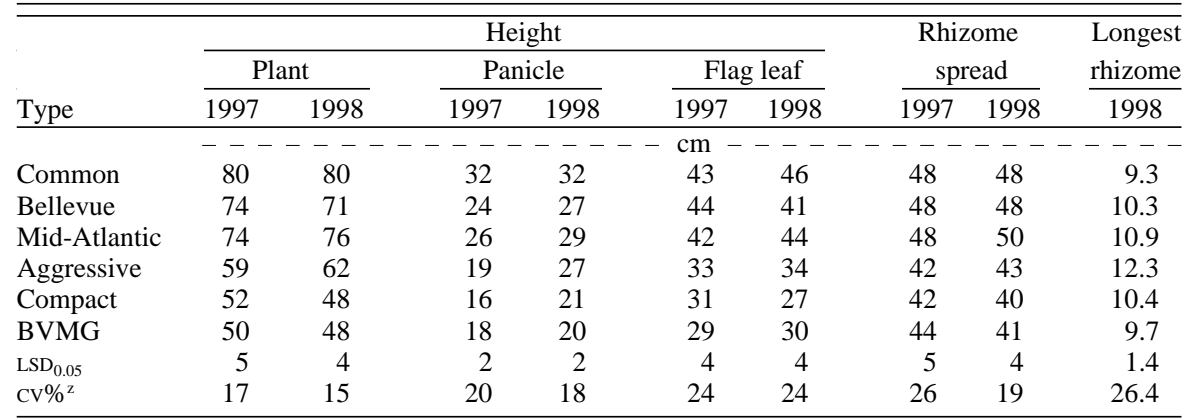

${ }^{\mathrm{z}}$ The $\mathrm{CV}$ presented is the total $\mathrm{CV}$ for the analysis of variance.

Table 3. Flag leaf length, subtending leaf length, and width of Kentucky bluegrass types established in a field trial in May 1996.

\begin{tabular}{|c|c|c|c|c|c|c|}
\hline \multirow[b]{2}{*}{ Type } & \multicolumn{2}{|c|}{ Flag leaf length } & \multicolumn{2}{|c|}{$\begin{array}{l}\text { Subtending } \\
\text { leaf length }\end{array}$} & \multicolumn{2}{|c|}{$\begin{array}{l}\text { Subtending } \\
\text { leaf width }\end{array}$} \\
\hline & 1997 & 1998 & 1997 & 1998 & 1997 & 1998 \\
\hline & --- & --- & --- & --- & --- & -- \\
\hline Mid-Atlantic & 6.6 & 4.1 & 9.4 & 8.0 & 5.8 & 3.7 \\
\hline Common & 5.5 & 4.1 & 8.6 & 7.4 & 4.6 & 2.7 \\
\hline Compact & 5.2 & 2.9 & 7.9 & 5.8 & 4.8 & 3.1 \\
\hline Bellevue & 5.1 & 3.1 & 8.3 & 6.5 & 5.9 & 3.8 \\
\hline BVMG & 4.9 & 3.2 & 6.9 & 5.9 & 5.2 & 3.1 \\
\hline Aggressive & 4.2 & 3.0 & 6.6 & 5.4 & 4.9 & 2.9 \\
\hline $\mathrm{LSD}_{0.05}$ & 0.9 & 0.6 & 1.0 & 0.8 & 0.3 & 0.4 \\
\hline $\mathrm{CV} \%^{\mathrm{z}}$ & 34.9 & 32.5 & 26.8 & 26.8 & 12.8 & 26.3 \\
\hline
\end{tabular}

${ }^{\mathrm{z}}$ The $\mathrm{CV}$ presented is the total $\mathrm{CV}$ for the analysis of variance. 
Table 4. Eigenvectors of the principal component axes (PC) from principal component analysis of Kentucky bluegrass types. Eigenvalues and their contribution to total variation are listed at the bottom of columns.

\begin{tabular}{|c|c|c|c|c|c|c|c|c|c|c|c|c|c|c|c|c|}
\hline \multirow[b]{2}{*}{ Character } & \multicolumn{2}{|c|}{$\mathrm{PC} 1$} & \multicolumn{2}{|c|}{$\mathrm{PC} 2$} & \multicolumn{2}{|c|}{ PC3 } & \multicolumn{2}{|c|}{ PC4 } & \multicolumn{2}{|c|}{ PC5 } & \multicolumn{2}{|c|}{ PC6 } & \multicolumn{2}{|c|}{ PC7 } & \multicolumn{2}{|c|}{ PC8 } \\
\hline & 1997 & 1998 & 1997 & 1998 & 1997 & 1998 & 1997 & 1998 & 1997 & 1998 & 1997 & 1998 & 1997 & 1998 & 1997 & 1998 \\
\hline & --- & --- & --- & --- & --- & - Eige & nvectors & --1 & --- & --- & --- & --1 & --- & --- & --- & --1 \\
\hline Plant height & 0.44 & 0.44 & -0.27 & -0.07 & -0.89 & 0.18 & -0.22 & -0.17 & -0.27 & 0.34 & -0.37 & -0.04 & -0.69 & 0.25 & --- & -0.75 \\
\hline Panicle length & 0.34 & 0.34 & -0.46 & 0.07 & -0.50 & 0.63 & -0.29 & -0.15 & 0.46 & -0.06 & 0.27 & 0.60 & 0.23 & -0.20 & --- & 0.25 \\
\hline Flag leaf height & 0.43 & 0.43 & -0.13 & -0.12 & 0.14 & -0.02 & -0.88 & -0.19 & -0.64 & 0.51 & -0.01 & -0.43 & 0.56 & 0.04 & --- & 0.56 \\
\hline Flag leaf length & 0.36 & 0.36 & 0.59 & -0.21 & -0.11 & -0.07 & -0.14 & 0.66 & 0.34 & -0.19 & -0.57 & 0.16 & 0.23 & 0.54 & --- & 0.16 \\
\hline Subtending leaf length & 0.38 & 0.41 & 0.53 & -0.15 & 0.08 & -0.23 & -0.20 & 0.36 & -0.06 & -0.07 & 0.68 & -0.07 & -0.25 & -0.77 & --- & -0.17 \\
\hline Subtending leaf width & 0.36 & 0.25 & 0.01 & 0.40 & -0.27 & -0.68 & 0.89 & -0.25 & 0.01 & 0.12 & 0.05 & 0.48 & -0.06 & 0.08 & --- & 0.01 \\
\hline Rhizome spread & 0.32 & 0.38 & -0.26 & 0.17 & 0.79 & -0.04 & 0.11 & -0.36 & 0.43 & -0.74 & 0.01 & -0.37 & 0.00 & 0.09 & --- & 0.01 \\
\hline Farthest ext. rhizome & --- & 0.04 & --- & 0.85 & --- & 0.22 & --- & 0.39 & --- & 0.14 & --- & -0.24 & --- & -0.02 & --- & -0.02 \\
\hline Eigenvalue & 4.50 & 4.60 & 1.06 & 1.14 & 0.63 & 0.94 & 0.46 & 0.67 & 0.27 & 0.32 & 0.04 & 0.23 & 0.01 & 0.10 & --- & 0.02 \\
\hline $\begin{array}{l}\text { Percentage of } \\
\text { total variation }\end{array}$ & 64.7 & 57.1 & 15.1 & 14.3 & 9.0 & 11.7 & 6.6 & 8.4 & 3.8 & 3.9 & 0.6 & 2.9 & 0.2 & 1.3 & --- & 0.2 \\
\hline
\end{tabular}

subtending leaf length (1997), and PC2 with farthest spreading rhizome and subtending leaf width (1998). Certain classification types (Common, Compact, Bellevue, Mid-Atlantic, and BVMG) formed distinctive clusters based on morphological measurements of spacedplants (Figs. 1 and 2).

This analysis also indicates the similarity in growth characteristics of the BVMG and Compact types, as well as of the Bellevue and Mid-Atlantic types, and shows that the BVMG and Compact are more similar in growth habit than reported earlier (Bara et al., 1993). However, distinct differences in other traits, not studied in this experiment, exist between these types. For example, the BVMG type cultivars are susceptible to stripe smut disease, while the Compact types are resistant (Bara et al., 1993; Murphy et al., 1997). Compact types are extremely resistant to leaf spot disease, while cultivars in the BVMG type are moderately susceptible (Bara et al., 1993; Murphy et al., 1997). Analysis by PCA also revealed the lack of uniformity within the Aggressive and Other types. Aggressive type cultivars did not form a distinct group based on the growth characteristics measured. This indicates that the Aggressive type cultivars are morphologically diverse except for the shared characteristics of high shoot density (not measured in this study) and aggressive lateral spread. Variability has also been observed within this group for other characteristics, including winter performance and disease resistance (Murphy et al., 1997).

\section{Conclusion}

Common, Bellevue, and Mid-Atlantic types have a more upright growth habit than the Compact and BVMG types. The differences in morphological characteristics observed may be an expression of adaptive characteristics that developed in response to the physical environment in which these genotypes evolved (Hay and Pedersen, 1986).

High-latitude plants persist under short days and low temperatures because induction of dormancy prevents frost damage during autumn (Hay and Pedersen, 1986). Hay and Pedersen (1986) found that cultivars of timothy (Phloem pratense L.) adapted to higher lati-

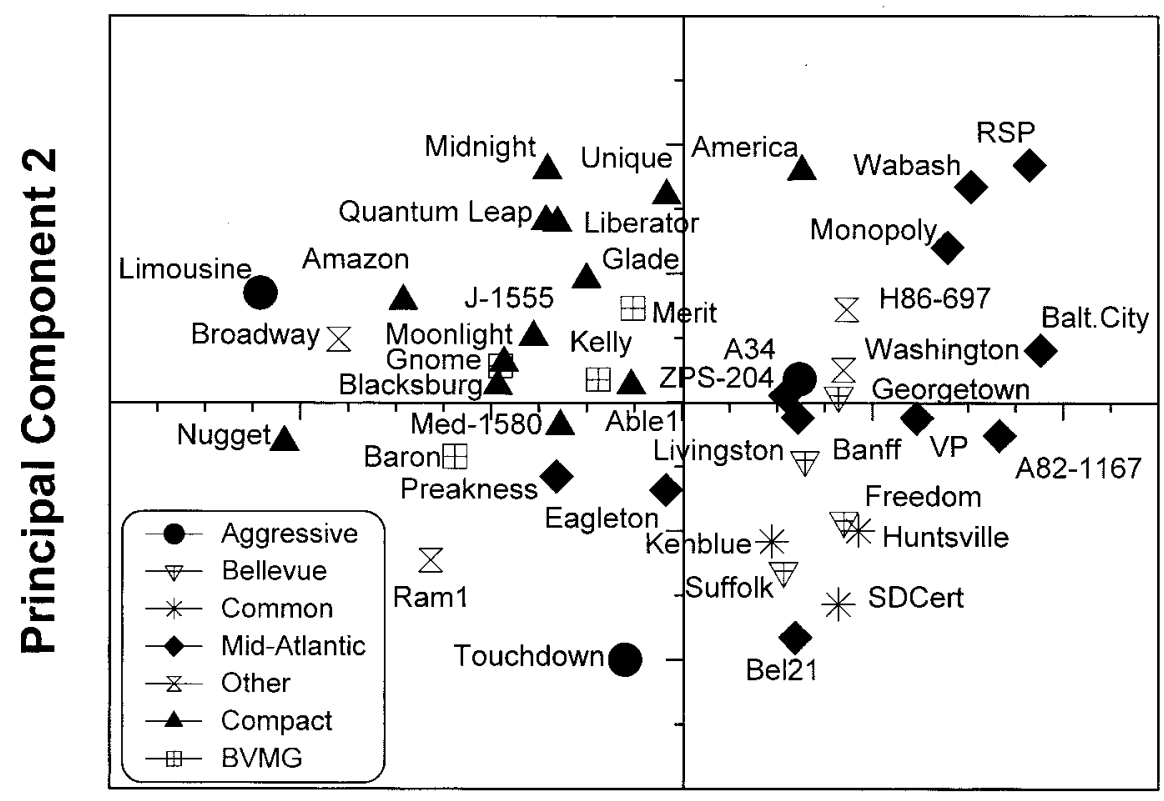

\section{Principal Component 1}

Fig. 1. Principal component analysis of Kentucky bluegrass cultivars and selections based on morphological characteristics in 1997. PC1 accounted for $64.7 \%$ of the variation and correlated with plant height, flag leaf height and subtending leaf length. PC 2 accounted for $15.1 \%$ of the variation and correlated with flag leaf length and subtending leaf length.

tudes had much slower growth rates during short days than did cultivars adapted to lower latitudes. Shoot growth was only stimulated under long photoperiods and low temperatures, an adaptation to the short growing season at high latitudes. These growth responses are indicative of the winter performance described by Murphy et al. (1997) and morphological characteristics (Table 2) of the Compact type Kentucky bluegrasses. Cultivars within the Compact type exhibit characteristics of plants adapted to higher latitudes and may need a more dramatic change in daylength before spring growth or leaf elongation is initiated.

Many cultivars and selections within the Common, Bellevue, and Mid-Atlantic types are adapted to New Jersey and the mid-Atlantic region $\left(37^{\circ}\right.$ to $42^{\circ} \mathrm{N}$ lat.). The mid-Atlantic region is characterized by shorter days and warmer temperatures during the growing season than those in regions of higher latitudes and cooler climates. Cultivars adapted to the mid-Atlantic region may green up earlier in the spring and their leaves may elongate more rapidly than other cultivars adapted to higher latitudes. These plants may be responding to less dramatic increases in temperature and daylength that are found in lower latitudes (Parsons and Robson, 1980).

Except for the Aggressive type, principal component analyses of the morphological data support the classification groups described by Bara et al. (1993) and Murphy et al. (1997), which were based on performance under mowing. Further development of this classification system is needed as more cultivars are developed with distinct characteristics. 


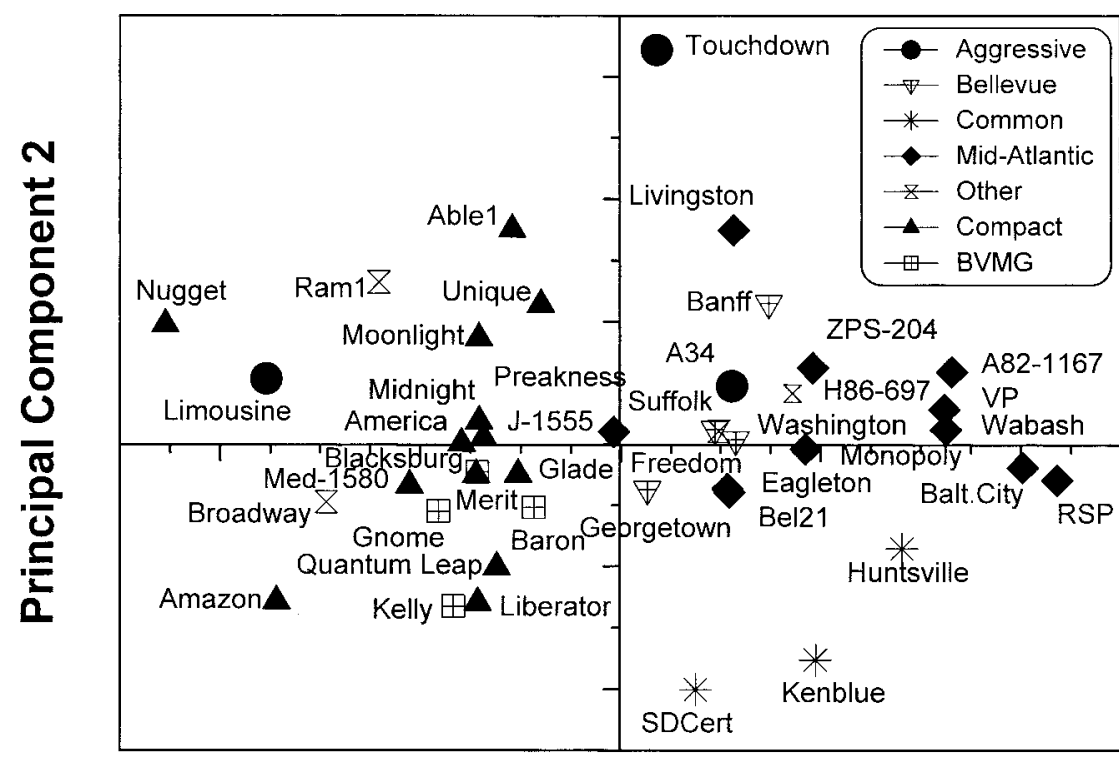

\section{Principal Component 1}

Fig. 2. Principal component analysis of Kentucky bluegrass cultivars and selections based on morphological characteristics in 1998. PC1 accounted for $57.1 \%$ of the variation and correlated with plant height, flag leaf height, and subtending leaf length. PC2 accounted for $14.3 \%$ of the variation and correlated with farthest spreading rhizome and subtending leaf width.

\section{Literature Cited}

Bara, R.F., W.K. Dickson, J.A. Murphy, D.A. Smith, and C.R. Funk. 1993. Performance of Kentucky bluegrass cultivars and selections in New Jersey turf trials. Rutgers Turfgrass Proc. 25:49-94.

Bashaw, E.C. and C.R. Funk. 1987. Apomictic grasses, p. 40-82. In: W.R. Fehr(ed.). Principles of cultivar development. Vol. 2. Crop species. Macmillan, New York.

Bonos, S.A. 1997. Evaluation of Kentucky bluegrass genotypes in response to summer stress. MS Thesis, Rutgers Univ., New Brunswick, N.J.

Burt, M.G. and N.E. Christians. 1990. Morphologi- cal and growth characteristics of low- and highmaintenance Kentucky bluegrass cultivars. Crop Sci. 30:1239-1243.

Ebdon, J.S., A.M. Petrovic, and S.J. Schwager. 1998. Evaluation of discriminant analysis in identification of low- and high-use Kentucky bluegrass cultivars. Crop Sci. 38:152-157.

Hay, R.K.M. and K. Pedersen. 1986. Influence of long photoperiods on the growth of timothy (Phloem pratense L.) varieties from different latitudes in northern Europe. Grass and Forage Sci. 41:311-317.

Iezzoni, A.F. and M.P. Pritts. 1991. Applications of principal component analysis to horticultural research. HortScience 26:334-338.

Meyer, W.A. and C.R. Funk. 1989. Progress and benefits to humanity from breeding cool-season grasses for turf, p. 31-48. In: D.A. Sleper, K.H. Asay, and J.F. Pedersen (eds.). Contributions from breeding forage and turf grasses. CSSA Spec. Publ. 15. Crop Sci. Soc. of Amer., Madison, Wis.

Morris, K.N. 1997. National Kentucky bluegrass test-1995: Progress report 1997, Natl. Turfgrass Evaluation Program. U.S. Dept. Agr., Agr. Res. Serv., Beltsville, Md.

Murphy, J.A., S.A. Bonos, and P. Perdomo. 1997. Classification of Poa pratensis genotypes. Intl. Turfgrass. Soc. Res. J. 8:1176-1183.

Parsons, A.J. and M.J. Robson. 1980. Seasonal changes in the physiology of S24 perennial ryegrass (Lolium perenne L.). 1. Response of leaf extension to temperature during transition from vegetative to reproductive growth. Ann. Bot. 46:435-444.

Pepin, G.W. and C.R. Funk. 1971. Intraspecific hybridization as a method of breeding Kentucky bluegrass (Poa pratensis L.) for turf. Crop Sci. 11:445-448.

SAS Institute. 1985. In: The princomp procedure, $\mathrm{p}$ 621-637. SAS users guide: Statistics, vers. $5^{\text {th }}$ ed. Ch. 28. SAS Inst., Cary, N.C. 